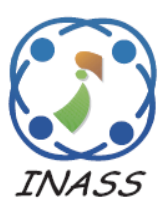

\title{
Medical Image Segmentation Based on Extreme Learning Machine Algorithm in Kernel Fuzzy C-Means Using Artificial Bee Colony Method
}

\author{
Hemalatha Kivudujogappa Lingappa ${ }^{1 *} \quad$ Hosahally Narayangowda Suresh ${ }^{2}$ \\ ${ }^{1}$ Reva University, India \\ ${ }^{2}$ Bangalore Institute of Technology, India \\ * Corresponding author’s Email: hema.sjcit@gmail.com
}

Sunil Kumar Manvi ${ }^{1}$

\begin{abstract}
In image segmentation field, the Fuzzy C-Means (FCM) algorithm is a well-known algorithm for its simplicity and membership function that can control the overlapped clusters effectively with a predefined number of clusters. Despite the fact, the standard FCM algorithm is noise sensitive. To solve the issue, we proposed a new method of clustering named Kernel Fuzzy C-means (KFCM) clustering. KFCM performed well in terms of clustering however, for pattern recognition KFCM has issues. The first one is grouping the similar objects in a single partition due to nonawareness of patterns and the second one is misclassification of data due to the standard structure of the membership subspace plane. Non-awareness of patterns of KFCM is solved by an Extreme Learning Machine (ELM) algorithm and Artificial Bee colony (ABC) algorithm utilized for optimizing the structure of the membership subspace plane. Experimental results showed that the proposed KFCM algorithm performed better segmentation for pattern recognition. At last effectiveness of the proposed algorithm has been evaluated based on comparing the K Means, FCM, spatial FCM, and KFCM algorithms in terms of centroids, segmentation accuracy, and pixel error. The proposed methodology improved the segmentation accuracy up to $0.8-5.5 \%$ compared to the existing methods.
\end{abstract}

Keywords: Artificial bee colony, Clustering, Extreme learning machine, Fuzzy c-means, Image segmentation, Kernel fuzzy c-means.

\section{Introduction}

Image segmentation is crucial research field since it has a vast amount of real world application, for example, robot vision, object recognition, geographical imaging and color imaging and medical imaging, etc. Generally, the major task of the image segmentation technique is to make the partitions of an image into non-overlapping, constituent regions based on the features such as gray level, color, tone, texture etc. [1-3]. Commonly, the image segmentation methodology is processed in four different ways, such as graph partitioning method [4], [5], model based technique [6], morphological strategies and clustering based technique [7, 8]. In the last decades, most of the segmentation research methodologies are done by the cluster-based technology. Among them, the fuzzy technique is a standard method and it can preserve more image info than the other clustering methodology $[9,10]$.

The membership value of every pixel of an image is assigned by the FCM on the basis of closeness of the image pixel to the centroid of each cluster, as the members of the similar partition have more similarity. The cluster centroids and the corresponding membership values are updated at each iteration, which leads the FCM algorithm classifies the image by generating the partitions of similar pixels in image space. FCM algorithm is efficient for image segmentation [11]. However, FCM in getting struggle to handle the noisy image, particularly when, there is no any prior knowledge of the noise [12]. While handling the noisy image, the FCM methodology failed to distinguish the pixels, which contain noise. The reason behind this is abnormal feature data, which is the main drawback of FCM. In order to solve this kind of problem we plan to utilize 
a kernel function to the fuzzy c means clustering algorithm (KFCM). The kernel function helps to formulate the distance between the two pixels that are available in the high dimensional feature space called kernel space. The kernel function converts the data in the image plane to high dimensional feature space. Non-linear separation problem in the image plane to linear separation problems in feature version is done by the mapping function, which leads to convert the feature space to kernel space clustering [13]. The KFCM algorithm also has the two drawbacks such as it is sensitive with respect to the initial centroid and secondly it can be struggled with the local minima. Furthermore, there is no previous information about the optimal parameters of the kernel functions that can be solved by the learning algorithm and the evolutionary algorithm.

Here, we propose an algorithm for efficient pattern recognition methodology through kernel fuzzy c means algorithm for segmentation process. Initially, the standard KFCM algorithm is applied to the extreme learning algorithm in order to learn the patterns of the images to avoid the similar objects in an image not group in a single partition because of the standard membership function. Next, we are incorporating the $\mathrm{ABC}$ algorithm to optimize the membership scaling function.

This paper is organized in the following order: the recent researches associated with the KFCM based segmentation are presented in the section 2 , and the section 3 comprises the objectives of the proposed algorithm. Section 4 describes the proposed KFCM algorithm for image segmentation. Section discussed about the experimental result finally the conclusion summed up into section 6 .

\section{Related works}

Numerous research techniques are suggested by researchers in medical image segmentation. In this scenario, a brief evaluation of some essential contributions to the existing literatures presented in this section.

G. Huang, S. Song, J.N. Gupta, and C. Wu, [14] extended ELMs for semi-supervised and unsupervised tasks based on the manifold regularization. The main benefits of the developed algorithm were: 1. Semi-supervised ELM (SS-ELM) and the Unsupervised ELM (US-ELM) showed learning ability and computational effectiveness of ELMs, 2. Both algorithms manages multiclass classification or multi-cluster clustering; 3. Both algorithms were inductive and managed unseen data at test time directly. Also, all the supervised, semisupervised, and unsupervised ELMs were suitable for unified framework. A broad experimentation with a wide range of data sets showed that the proposed algorithm was better than the other semi-supervised or unsupervised learning algorithms with regard to correctness and effectiveness. This action gave a new viewpoint to understand the procedure of random feature mapping, which was the main thought in ELM theory. The learning performance of the subsequent classification models were affected by the noise or redundant features.

H. Li, H. He, and Y. Wen, [15] presented an algorithm for image segmentation, called Dynamic Particle Swarm Optimization and K-means clustering algorithm (DPSOK). Dynamic Particle Swarm Optimization (DPSO) and K-means clustering method was the base of DPSOK algorithm. It makes DPSOK a balanced optimized capable algorithm by enhancing the computing technique of its inertia weight and learning factors. To provide balance optimization ability in DPSOK, the developed approach improved the calculation of its inertia weight and learning factors. Experimentation outcomes showed that DPSOK algorithm efficiently enhance the K-means algorithm's global search ability. DPSOK algorithm delivered better results in enhancing image segmentation quality and efficiency compared to conventional PSO K-means algorithm. In a few cases, the training data were dependent evaluation or semi-supervised adjustment, which was needed to be automated.

S. Pereira, A. Pinto, V. Alves, and C.A. Silva, [16] proposed a Convolutional Neural Networks (CNN) for automatic brain tumour detection and segmentation. Here, the CNN method was developed by small kernels (fewer number of weights) in the network. Along with $\mathrm{CNN}$ method, the intensity normalization was accomplished for brain tumour segmentation. The proposed method delivers an effective brain tumour segmentation in the spatial localization and structural composition. The experiment was carried-out on publicly available database: BRATS 2013 in order to validate the proposed method accuracy, robustness and speed. The developed methodology would fail, when the growth of the tumour cell boundaries were constrained.

Y. Megersa, and G. Alemu, [17] developed an automatic brain tumour detection and segmentation framework based on skull stripping and Fuzzy Hopfield Neural Networks (FHNN) methodology for detecting the brain tumour tissues. The advantage of proposed methodology was fully automated, there was no user intervention for segmenting the normal and tumour tissues, so the time consumption of detecting the tumour cell was very less. Extensive 
experiments were carried out on T1-w and T2-w head Magnetic Resonance Imaging (MRI) brain images for demonstrating the robustness of proposed scheme. The disadvantage of FHNN methodology was more complex to find the projection of free space. Free space can able to provide better discriminant ability in feature extraction.

Y. Song, Z. Ji, Q. Sun, and Y. Zheng, [18] proposed a new level set based model for tumour segmentation from multi-modality MRI. Initially, detect the abnormal regions using a region based active contour model. Then, a level set formulation was applied locally to approximate the image intensities on two sides of the contour. The developed methodology significantly improves the computational performance and the level set algorithm delivers high accuracy of brain tumour segmentation in multidimensional image data. The developed model improves the brain segmentation accuracy significantly, but it was very time consuming, since it computes the neighbourhood term in each iteration step.

An unsupervised algorithm is implemented to overcome the above mentioned drawbacks and additionally enhance the performance of medical image segmentation.

\section{Proposed KFCM algorithm for efficient segmentation}

In this paper, we propose an algorithm for efficient pattern recognition methodology through kernel fuzzy c means algorithm for segmentation process. Initially, the standard KFCM algorithm is applied to the extreme learning algorithm in order to learn the patterns of the images to avoid the similar objects in an image not group in a single partition because of the standard membership function. Next, we are incorporating the $\mathrm{ABC}$ algorithm to optimize the membership scaling function with the intention of deriving the suitable structure of the membership subspace plane.

\subsection{Fuzzy c-means algorithm}

Fuzzy c means algorithm is partitioning the dataset $\left\{x_{k}\right\}_{k=1}^{N}$ into $c$ number of clusters based on the following objective function in Eq. (1).

$$
J_{m}=\sum_{i=1}^{c} \sum_{k=1}^{N} u_{i k}^{p}\left\|x_{k}-v_{i}\right\|^{2}
$$

Where, $p$ indicates the real number, which denotes the quantity controlling of the fuzziness of the resultant group, $u_{i k}^{p}$ is the membership of the data point $x_{k}$ belongs to the cluster $i$ and is the $x_{k}$ pixel of the image which satisfying $\sum_{i=1}^{c} u_{i k}=1$ and $v_{i}$ is the centroid of the cluster. From the above Eq. (1), where $c$ is the total number of clusters and $N$ denotes the number of data points. The FCM makes the partitioning by iteratively updating the membership values and the cluster centroids. The membership value of each data point to the every centroid also derived after each time updating of centroids that is done by the Eq. (2).

$$
u_{i k}=\frac{1}{\sum_{j=1}^{c}\left(\frac{\left\|x_{k}-v_{i}\right\|^{2}}{\left\|x_{k}-v_{j}\right\|^{2}}\right)^{1 / p-1}}
$$

The cluster centroids are updated based on the distance between the data point to the cluster centroid which is done by the Eq. (3).

$$
v_{k}=\frac{\sum_{k=1}^{N} x_{k} U_{i k}^{p}}{\sum_{k=1}^{N} U_{i k}^{p}}
$$

The objective function performs the calculation to measure the weighted sum of results between the cluster center and data presents in the fuzzy clusters.

FCM provides better segmentation results for the images, which does not have any noise. However, the FCM fails to classify the noisy data because of the irregularities of the feature data, which leads to assign the membership values become erroneous. This is the main reason for improper segmentation occurs during the processing of a noisy image by the FCM.

\subsection{Kernel fuzzy c-means (KFCM)}

With the intention to overcome the problem of the standard FCM algorithm, the KFCM algorithm is designed. With the aid of a nonlinear mapping function, the KFCM converts the input data in the image plane into higher dimensional feature space. The complex and nonlinear separable problem in the input plane can be converted with the aid of the mapping function into linearly separable in the future space. Then, the FCM can perform its operation with the derived feature space. The objective function of the KFCM is presented in the following Eq. (4).

$$
\begin{gathered}
J_{m}=\sum_{i=1}^{c} \sum_{k=1}^{N} u_{i k}^{p}\left\|\varphi\left(x_{k}\right)-\varphi\left(v_{i}\right)\right\|^{2}= \\
2 \sum_{i=1}^{c} \sum_{k=1}^{N} u_{i k}^{p}\left(1-k\left(x_{k}-v_{i}\right)\right)
\end{gathered}
$$

Where, $\varphi$ is the mapping function. Here we use the Gaussian kernel function for non-linear mapping of the image plane into the linear high dimensional feature space. The Gaussian kernel function is presented in the Eq. (5). 


$$
K=K(x, y)=\exp \left(-\frac{d(x, y)^{2}}{\sigma^{2}}\right)
$$

Substituting Eq. (5) into the Eq. (4) then the Gaussian kernel objective function is presented in the Eq. (6).

$$
\begin{gathered}
J_{m}=\sum_{i=1}^{c} \sum_{k=1}^{N} u_{i k}^{p}\left\|\varphi\left(x_{k}\right)-\varphi\left(v_{i}\right)\right\|^{2}= \\
2 \sum_{i=1}^{c} \sum_{k=1}^{N} u_{i k}^{p}\left(1-K\left(x_{k}-v_{i}\right)\right)
\end{gathered}
$$

Where, the membership function and the updating the centroid is done by using the Eqs. (7) and (8), respectively.

$$
\begin{aligned}
& u_{i k}=\frac{\left(1-K\left(x_{k}, v_{i}\right)\right)^{\frac{-1}{(p-1)}}}{\sum_{i=1}^{c}\left(1-K\left(x_{j}, c_{i}\right)\right)^{\frac{-1}{(p-1)}}} \\
& v_{i}=\frac{\sum_{k=1}^{n} u_{i k}^{p} K\left(x_{k}-v_{i}\right) x_{k}}{\sum_{k=1}^{n} u_{i k}^{p} K\left(x_{k}, v_{i}\right)}
\end{aligned}
$$

\subsection{Extreme learning machine}

The mapping function of KFCM makes the input data into high dimensional feature vector, which helps the FCM to handling the noisy image. However, the processing with the high dimensional feature vectors demands a number of iterations, which leads more execution time to make the membership function suitable for image segmentation. Due to the standard membership function, the similar objects in an image not grouped in a single partition. To solve this problem, we utilize the extreme learning algorithm, which helps to make the membership function based on patterns in the image by learning methodology.

The extreme learning machines are used in the artificial neural network for training the network. In this paper, we adapt the learning method to predict the future value of the fuzzy membership value, which directs to decrease the running time for the clustering process. The Eq. (9) is used to predict the future value of the fuzzy membership values.

$$
u_{\hat{i k}}=\eta\left(\frac{1}{\left(u_{i k} X\right)}\right) Y
$$

Where, $u_{i k}$ represents the future membership matrix and $u_{i k}$ represents the existence membership matrix. The learning rate is represented as $\eta, X$ indicates the input matrix and $Y$ specifies current partitioned matrix. The future centroid is calculated by using the Eq. (10).

$$
v_{i}^{\hat{i}}=\frac{\sum_{k=1}^{n} u_{\hat{i} k} K\left(x_{k}-v_{i}\right) x_{k}}{\sum_{k=1}^{n} u_{i k}^{\hat{k}} K\left(x_{k}, v_{i}\right)}
$$

\subsection{Artificial bee colony}

In the standard structure of the membership function, false acceptance ratio and false rejection ratio values are more due to the persistent weight value of the membership function. In our approach, the structure of the membership subspace plane is decided by $\mathrm{ABC}$ algorithm by optimizing the weight value of the membership function. The $A B C$ algorithm is a swarm intelligence algorithm based on the foraging behaviour of honey bee colonies. Here, an $\mathrm{ABC}$ comprises three groups: employed bees, onlookers and scouts. Employed artificial bees represent the first half of the colony, while the second half consist of the onlookers. For each food source there is one employed bee. In $\mathrm{ABC}$, the population consists of food sources, where each food source represents a potential solution to the problem at hand. Each solution is associated with a nectar amount of the food source, which represents the fitness value of the solution.

Step 1: Initialization: An initial population of solutions is generated. NS represents the number of solutions; it is the number of the employed bees and the onlookers. The number of elements of these vectors is NS, which represents the number of grey levels of the input image. The $\mathrm{ABC}$ generates a randomly distributed initial population of NS solutions (food source positions). In this paper, the initial population takes as set of membership scaling function values $\alpha_{i},(0 \leq \alpha \geq 1),(1 \leq i \geq N S)$. The bee creates a new solution from a randomly chosen one, which is displayed in the Eq. (11).

$$
\begin{array}{r}
\quad S_{1} \rightarrow \alpha_{1}, S_{2} \rightarrow \alpha_{2}, S_{i} \rightarrow \alpha_{i}, S_{N S-1} \rightarrow \alpha_{N S-1}, \\
S_{N S} \rightarrow \alpha_{N S}
\end{array}
$$

After this initialization, the algorithm repeats the following steps cyclically until a stop condition is met. In this work, the stop condition has been chosen to be a predefined number of iterations.

Step 2: Each solution in the population is evaluated by calculating its fitness function, from which $\mathrm{N}$ number of best solutions in the population are stored. In this paper, the fitness value of the solution (membership scaling function) is evaluated through calculating the weight value of membership functions, that can be obtained through the following Eq. (12).

$$
M F_{W}^{i}=\left(1-\alpha_{i}\right) \times\left[(\text { gauss } M F)\left(d-v_{j}\right)\right]
$$


From the above Eq. (12), where d indicates the vector value of pixel $x$ and the gauss MF function represents Gaussian membership function.

The Employed Bee Stage: During each iteration of the algorithm, each employed bee determines a new food source $\alpha_{i}^{k+1}$ in the neighbourhood of its currently associated food source $\alpha_{i}^{k}$.

Step 3: A new solution is generated from each old solution, which is given in the Eq. (13).

$$
\alpha_{i}^{k+1}=\alpha_{i}^{k}+\varphi_{q}\left(\alpha_{i}^{k}-\alpha_{B}^{k}\right)
$$

Where, $\varphi_{q}$ is a random number the range of random number is $\varphi_{q} \in[-1,1]$ and $\alpha_{i}^{k}$ is represents $i^{\text {th }}$ solution of $k^{\text {th }}$ iteration and $\alpha_{B}^{k}$ represents the best solution of $k^{t h}$ iteration and $\alpha_{i}^{k+1}$ represents the updated solution.

Step 4: Each updated solution in the population is evaluated by calculating its fitness function, and which is compared with the existing solution whether the updated solution gives better nectar's amount or not. If the updated solution gives the better nectar's amount than the existing solution, then we take the updated solution, as we keep process with the existing solution. This is done by greedy selection approach. After the greedy selection process, the set of solutions is transferred to the onlooker bee.

The Onlooker's Bee Stage: After all, employed bees have completed their work, as described above; they share the nectar information of food sources with the onlookers. Consequently, each onlooker bee selects probabilistically a food source according to its nectar amount using the roulette wheel selection method. The probability $P_{i}$. with which a food source is selected by an onlooker is given in the Eq. (14).

$$
P_{i}=\frac{M F_{W}^{i}}{\sum_{i=1}^{N} M F_{W}^{i}}
$$

Where, $M F_{W}^{i}$ indicates the fitness value of the $i^{t h}$ solution and $N$ represents the total number of solution. The best solution can be taken based on the probability value of the solution $P_{i}$. If the solution cannot be improved over a particular number of iterations, then corresponding solution is considered as inactive solution that can be replaced by scout bee.

The Scout Bee Stage: If the food source cannot be improved for a set number of cycles, it is abandoned and replaced with a new food source by a scout. The Eq. (15) used for discovering the new solution.

$$
\alpha_{i}=l b+\operatorname{rand}(0,1) \times(u b-l b)
$$

Where, $l b$ and $u b$ represents the lower bound and upper bound, respectively.

The updating process is repeated up to $N$ number of iterations. The result of the final iteration produces the optimizing the weight value of the membership function $\left(\alpha_{i}\right)$ with the help of that the membership function structure can be derived. Now, our proposed ABC-ELM-KFCM produces better segmentation results than the existing segmentation results. The pattern recognition can be applied to the resultant segmented image obtained by the proposed ABCELM-KFCM algorithm for better recognition.

\section{Result and discussion}

For experimental simulation, MATLAB (version 2015a) was employed on PC with $3.2 \mathrm{GHz}$ with i5 processor. In order to estimate the efficiency of proposed algorithm, the performance of the proposed method was compared with FCM, k-means clustering, and Spatial-FCM (SFCM)-Modified Centroid Weight Particle Swarm Optimization (MPSO) [19] on the reputed database: BraTS. The performance of the proposed methodology was compared in terms of segmentation accuracy and pixel error.

\subsection{Performance measure}

Performance measure is defined as the relationship between input and output variables of a system understood by employing a suitable performance metric like segmentation accuracy. The general formula of accuracy is denoted in the Eq. (16).

$$
\text { Accuracy }=\frac{T P+T N}{T P+T N+F P+F N} \times 100
$$

Where, $T P$ is represented as true positive, $F P$ is denoted as false positive, $T N$ is stated as true negative and $F N$ is specified as false negative.

\subsection{Experimental analysis}

In this section, the proposed segmentation methodology (ABC-ELM-KFCM) is evaluated by considering a sample medical image from BraTS dataset. The effectiveness of the proposed algorithm is verified by comparing with a few existing algorithms like k-means, FCM and SFCM-MPSO algorithm [19]. K.L. Hemalatha, S. Ranjitha, and H.N. Suresh, [19] proposed spatial FCM clustering algorithm with modified centroid weight particle swarm optimization for image segmentation. In this research paper, the spatial function was summed with membership function in neighbourhood pixels. This action highly improves the effectiveness of 
segmentation. The simulation outcome shows that the developed methodology achieved better segmentation performance in terms of average error and cluster centroid. By analysing the Figs. 1-5, we conclude that the proposed methodology outperformed in terms of segmentation accuracy and pixel error.

The optimal segmentation of the medical image depends on the structure of membership plane of kernel fuzzy clustering algorithm that is done by optimizing the scaling function of membership. Optimizing the membership scaling function leads to get the better clustering formation, which is done, based on the ABC algorithm. Moreover, the clusters should be formed as per the patterns presented in an image, which guides to make exact size of segmentation area and helps to segregate the borders of each part of an image. Size of portions of an image and segregation of borders leads to build the better segmentation accuracy; the extreme learning algorithm was used in the membership function to learn the patterns of images.

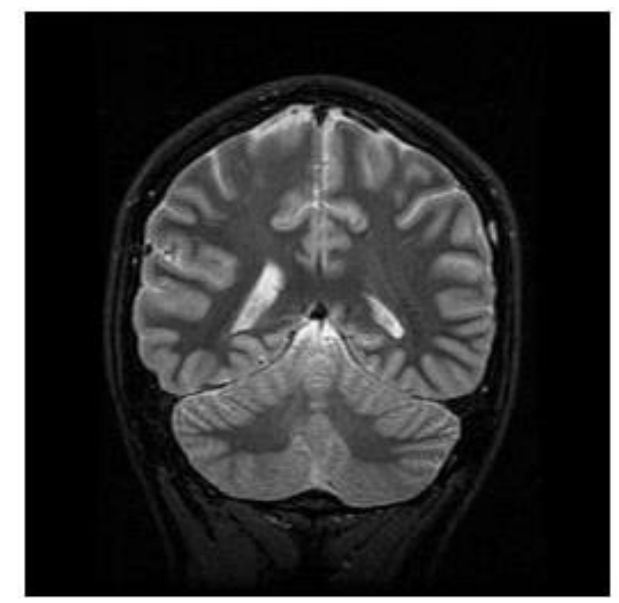

Figure. 1 Input brain image

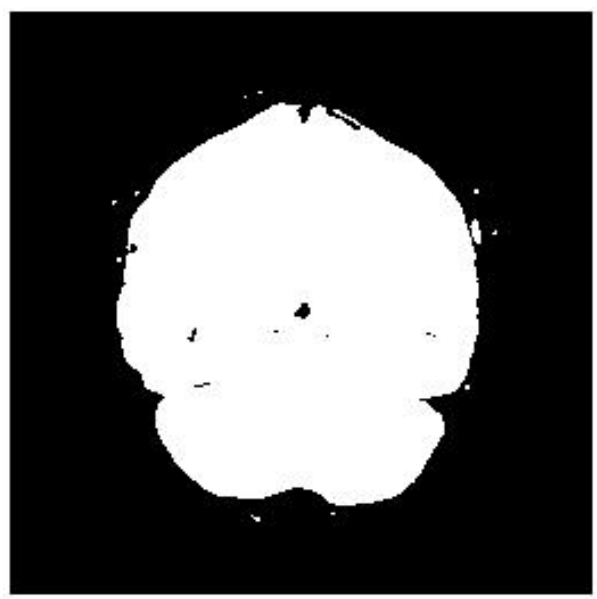

Figure. 2 K Means Clustering

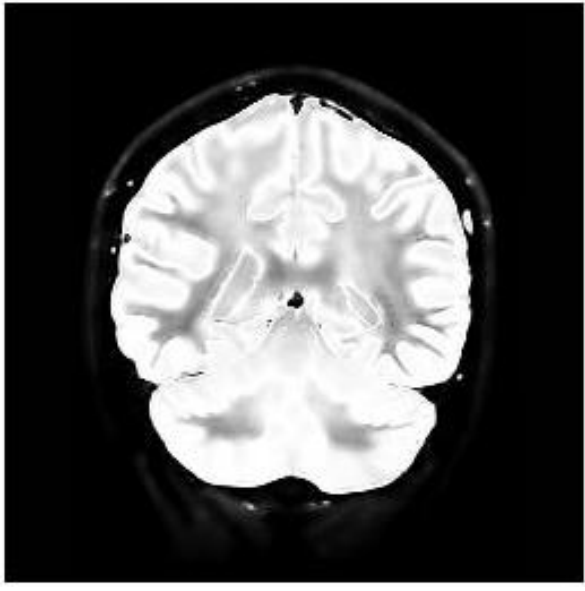

Figure. 3 FCM Clustering

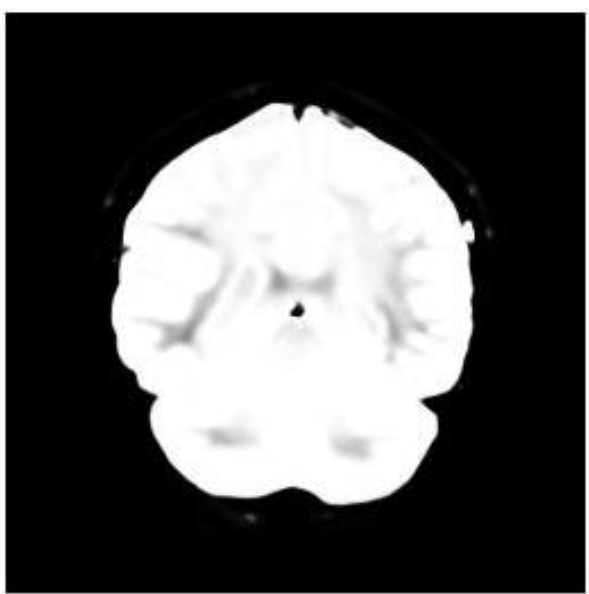

Figure. 4 SFCM-MPSO Clustering

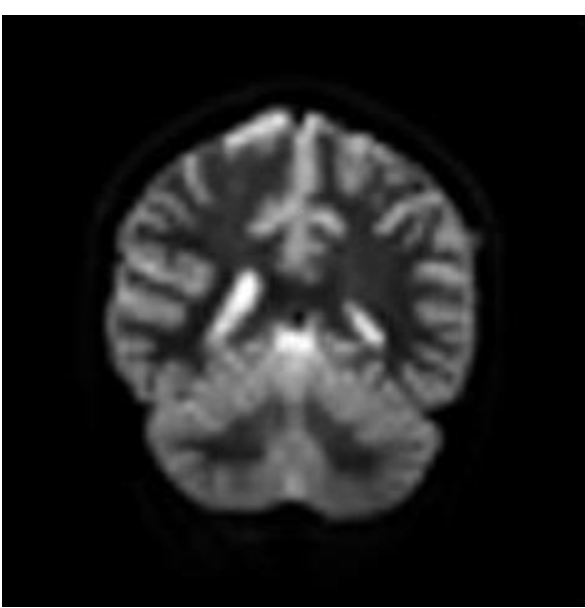

Figure. 5 Proposed ACO-ELM-KFCM

The proposed segmentation methodology (ABCELM-KFCM) is evaluated by comparing with existing segmentation algorithms such as K Means, FCM, KFCM and SFCM-MPSO [19] in terms of segmentation accuracy and pixel error. For experimental analysis, we considered both medical and multi-media images. 
Table 1. Comparison analysis of segmentation accuracy for medical images

\begin{tabular}{|c|c|c|c|c|c|}
\hline \multirow[t]{2}{*}{ Methods } & \multicolumn{4}{|c|}{ Segmentation Accuracy (\%) } & \multirow{2}{*}{$\begin{array}{c}\text { Average } \\
\text { Accuracy } \\
(\%)\end{array}$} \\
\hline & Centroids & Cluster 2 & Centroids & Cluster 3 & \\
\hline K-Means & $6.6254,98.6115$ & 1195 & $6.4421,77.6890,125.2745$ & 1201 & 91.68 \\
\hline FCM & $6.7935,101.1125$ & 976 & $6.154,76.2868,123.0570$ & 987 & 93.18 \\
\hline KFCM & $6.2517,99.9285$ & 522 & $121.5948,70.2040,4.3013$ & 548 & 96.28 \\
\hline $\begin{array}{c}\text { SFCM-MPSO } \\
{[19]}\end{array}$ & $6.7106,99.1261$ & 437 & $76.2868,6.1541,123.0570$ & 440 & 96.95 \\
\hline Proposed & $6.2476,99.9264$ & 424 & $121.5908,70.1984,4.2976$ & 429 & 97.03 \\
\hline
\end{tabular}

Table 2. Comparison analysis of pixel error for multimedia images

\begin{tabular}{|c|c|c|c|c|c|}
\hline \multirow{2}{*}{ Methods } & \multicolumn{4}{|c|}{ Segmentation Accuracy (\%) } & $\begin{array}{c}\text { Average } \\
\text { Accuracy } \\
(\%)\end{array}$ \\
\cline { 2 - 6 } & Centroids & Cluster 2 & Centroids & Cluster 3 & 75.73 \\
\hline K-Means & $151.4870,25.5933$ & 63655 & $121.0076,167.7719,22.3747$ & 63667 & 77.40 \\
\hline FCM & $153.6055,25.0161$ & 59223 & $122.3355,168.4849,20.4672$ & 59238 & 50.18 \\
\hline KFCM & $23.5953,154.3047$ & 51236 & $18.3111,168.1686,121.3009$ & 52656 & 81.40 \\
\hline $\begin{array}{c}\text { SFCM-MPSO } \\
{[19]}\end{array}$ & $153.6055,25.0161$ & 48744 & $118.1560,167.5352,21.7026$ & 48751 & 84.45 \\
\hline Proposed & $23.5943,154.2998$ & 40589 & $18.3012,168.1643,121.2986$ & 40899 & 8 \\
\hline
\end{tabular}

Table 3. Comparative analysis of proposed and exiting method

\begin{tabular}{|c|c|c|c|}
\hline References & Dataset & Segmentation approach & Accuracy (\%) \\
\hline $\begin{array}{c}\text {.H. Ali, M. Elmogy, E. } \\
\text { El-Daydamony, and A. } \\
\text { Atwan, [20] }\end{array}$ & BraTS & $\begin{array}{c}\text { Morphological pyramid with } \\
\text { FCM clustering method }\end{array}$ & 95.853 \\
\hline $\begin{array}{c}\text { G. Latif, D.A. Iskandar, } \\
\text { A. Jaffar, and M.M. Butt, } \\
\text { [21] }\end{array}$ & BraTS & KNN-DCT & 93.22 \\
\hline Proposed & BraTS & ABC-ELM-KFCM & 97.03 \\
\hline
\end{tabular}

By analysing the Tables 1 and 2, we conclude that our proposed methodology outperformed in terms of centroid optimization and segmentation accuracy and pixel error for medical image and multi-media image. Moreover, the multimedia images are produced less result when compared with the medical image, the reason behind this is not able to segregate the background and foreground accurately. The proposed methodology concentrated on designing the optimal structure of the membership plan by optimizing the membership scaling function value through the $\mathrm{ABC}$ algorithm. The main reason for increasing the accuracy is implementing the extreme learning algorithm into the membership function to learn the patterns of images. Learning the membership function of an image and optimizing the structure of the membership scaling function made our proposed algorithm become the enhanced version of KFCM.

\subsection{Comparative analysis}

Table 3 represents the comparative study of existing and the proposed work performance. H. Ali, M. Elmogy, E. El-Daydamony, and A. Atwan, [20] proposed a clustering approach: morphological pyramid with FCM clustering technique for automatic brain tumour segmentation. Here, a wavelet multi-resolution was used for maintaining the spatial context between the pixels. Then, morphological pyramid was employed for fusing the resultant multi-resolution brain images with the original image for improving the sharpness and also to decrease noise in the processed image. The experiment was carried-out on a publicly available database: BraTS in order to validate the proposed method accuracy. The developed methodology achieved $95.853 \%$ of classification accuracy. 
In addition, G. Latif, D.A. Iskandar, A. Jaffar, and M.M. Butt, [21] developed a supervised binomial classification methodology: K-Nearest Neighbour (KNN) for classifying the tumour and non-tumour MRI images by extracting the features of Discrete Cosine Transform (DCT). Then, segment the tumour region by using the image features along with actual image features. The experimental results demonstrated that the developed methodology achieved $93.22 \%$ of segmentation accuracy. Whereas, the proposed work achieves $97.03 \%$ of accuracy that was higher than the existing works.

\section{Conclusion}

With the intention of increasing, the performance and reducing the difficulties of KFCM, we proposed new segmentation methodology ABC-ELM-KFCM. The standard KFCM has some issues related to the membership function. The first one is misclassification of patterns present in an image, which was solved by implementing extreme learning machine into the membership function of KFCM algorithm. The next one is the standard structure of the membership subspace plane of an image, which causes grouping the similar objects in a single partition due to non-awareness of patterns. The optimal structure of the membership subspace plane of an image is derived from the $\mathrm{ABC}$ algorithm through optimizing the membership scaling function. The proposed segmentation methodology generates better segmentation results for pattern recognition and effectiveness of proposed algorithm was evaluated based on comparing the K Means, FCM, KFCM and SFCM-MPSO algorithms in terms of centroids, segmentation accuracy, and pixel error. Compared to other obtainable approaches in medical image segmentation, the proposed methodology showed $0.8-5.5 \%$ of enhancement in segmentation accuracy than the existing methods. In the future work, for classifying the classes of segmented brain region, descriptor level feature extraction methodologies will be employed with an appropriate multi-objective classification method.

\section{References}

[1] Y. Yong, Z. Chongxun, and L. Pan, "A novel fuzzy C-Means clustering algorithm for image thresholding", Measurement Science Review, Vol.4, No.1, pp.11-19 2004.

[2] M.R. Ferreira and F.D.A. De Carvalho, "Kernel fuzzy-c-means with automatic variable weighting", Fuzzy Sets and Systems, Vol.237, pp.1-46, 2014.
[3] S.R. Kannan, S. Ramathilagam, A. Sathya, and R. Pandiyarajan, "Effective fuzzy c-means based kernel function in segmenting medical images", Computers in Biology and Medicine, Vol.40, No.6, pp.572-579, 2010.

[4] J. Feng, L.C. Jiao, X. Zhang, M. Gong, and T. Sun, "Robust non-local fuzzy c-means algorithm with edge preservation for SAR image segmentation", Signal Process, Vol.93, No.2, pp.487-499, 2013.

[5] J. Shi and J. Malik, "Normalized cuts and image segmentation", IEEE Trans. Pattern Anal. Mach. Intell., Vol.22, No.8, pp.888-905, 2000.

[6] X. Descombes, M. Moctezuma, H. Maître, and J.P. Rudant, "Coastline detection by a Markovian segmentation on SAR images", Signal Process, Vol.55, No.1, pp.123-132, 1996.

[7] X. Zhang, L. Jiao, F. Liu, L. Bo, and M. Gong, "Spectral clustering ensemble applied to texture features for SAR image segmentation", IEEE Trans. Geosci. Remote Sens., Vol.46, No.7, pp.2126-2136, 2008.

[8] D. Xiang, T. Tang, C. Hu, Y. Li, and Y. Su, “A Kernel Clustering Algorithm with Fuzzy Factor: Application to SAR Image Segmentation", IEEE Geoscience and remote sensing letters, Vol.11, No.7, pp.1290-1294, 2014.

[9] B.N. Li, C.K. Chui, S. Chang, and S.H. Ong, "Integrating spatial fuzzy clustering with level set methods for automated medical image segmentation", Computers in Biology and Medicine, Vol.41, No.1, pp.1-10, 2011.

[10] M. Gong, Y. Liang, J. Shi, W. Ma, and J. Ma, "Fuzzy c-means clustering with local information and kernel metric for image segmentation", IEEE Transactions on Image Processing, Vol.22, No.2, pp.573-584, 2013.

[11] M. Gong, Y. Liang, J. Shi, W. Ma, and J. Ma, "Fuzzy C-means Clustering with Local Information and Kernel Metric for Image Segmentation", IEEE Transaction on Image Processing, Vol.22, pp.573-584, 2013.

[12] M. Yambal and H. Gupta, "Image Segmentation using Fuzzy C Means Clustering: A survey", International Journal of Advanced Research in Computer and Communication Engineering, Vol.2, No.7, 2013.

[13] S.M. Mahajan, and Y.K. Dubey, "Color Image Segmentation Using Kernalized Fuzzy C-means Clustering", In: Proc. of the Fifth International Conference on Communication Systems and Network Technologies, pp.1142-1146, 2015.

[14] G. Huang, S. Song, J.N. Gupta, and C. Wu, "Semi-Supervised and Unsupervised Extreme Learning Machines", IEEE Transactions on 
Cybernetics, Vol.44, No.12, pp. 2405-2417, 2014.

[15] H. Li, H. He, and Y. Wen, "Dynamic particle swarm optimization and K-means clustering algorithm for image segmentation", Optik International Journal for Light and Electron Optics, Vol.126, No.24, pp.4817-4822, 2015.

[16] S. Pereira, A. Pinto, V. Alves, and C.A. Silva, "Brain tumour segmentation using convolutional neural networks in MRI images", IEEE Transactions on Medical Imaging, Vol.35, No.5, pp. 1240-1251, 2016.

[17] Y. Megersa and G. Alemu, "Brain tumour detection and segmentation using hybrid intelligent algorithms", In: Proc. of International Conference on AFRICON, pp.1-8, 2015.

[18] Y. Song, Z. Ji, Q. Sun, and Y. Zheng, "A Novel Brain Tumor Segmentation from MultiModality MRI via A Level-Set-Based Model", Journal of Signal Processing Systems, Vol.87, No.2, pp.249-57, 2017.

[19] K.L. Hemalatha, S. Ranjitha, and H.N. Suresh, "Image segmentation based on modified centroid weight particle swarm optimization and spatial fuzzy C-means clustering algorithm", In: Proc. of the Applied and Theoretical Computing and Communication Technology, International Conference on IEEE, pp.723-727, 2015.

[20] H. Ali, M. Elmogy, E. El-Daydamony, and A. Atwan, "Multi-resolution MRI brain image segmentation based on morphological pyramid and fuzzy c-mean clustering", Arabian Journal for Science and Engineering, Vol.40, No.11, pp.3173-3185, 2015.

[21] G. Latif, D.A. Iskandar, A. Jaffar, and M.M. Butt, "Multimodal Brain Tumor Segmentation using Neighboring Image Features", Journal of Telecommunication, Electronic and Computer Engineering, Vol.9, No.2-9, pp.37-42, 2017. 